\title{
Challenges in the virtual assessment of COVID-19 infections in the community
}

Warren J. Mclsaac MD MSc CCFP Ross Upshur MD MSc MCFP FRCPC

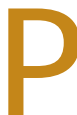

neumonia is a major complication of COVID-19 infection that can lead to respiratory failure, ICU admission, or death. While $81 \%$ of COVID-19 cases are considered mild, ${ }^{1}$ this figure includes cases of pneumonia that were considered non-severe based on definitions found in an American Thoracic Society (ATS)/Infectious Diseases Society of America (IDSA) guideline. ${ }^{2}$ Their definition of severe pneumonia is pneumonia with respiratory failure, organ failure, or septic shock or pneumonia with 3 minor criteria (eg, respiratory rate 30 or higher, hypotension requiring fluid resuscitation). ${ }^{2}$ Thus, mild pneumonia can still be a serious illness. Of more than 1000 patients with pneumonia due to COVID-19 admitted to Chinese hospitals, $84 \%$ met this definition of mild illness. ${ }^{3}$

Family physicians are being encouraged to provide virtual care for patients with COVID-19.4,5 How to determine which patients have mild cases of COVID-19 and are appropriate for care in the community during virtual assessments is unclear. ${ }^{6}$ This article will review recent findings to highlight challenges family physicians may face in providing virtual assessments for adults with suspected COVID-19 infections.

What is considered a "mild" COVID-19 infection? Most studies of patients with COVID-19 have been limited to those who have been hospitalized. ${ }^{3,7}$ Information regarding milder infections is limited. A US Centers for Disease Control and Prevention (CDC) report indicated that of 2449 patients with COVID-19, 21\% to $31 \%$ were hospitalized, $5 \%$ to $12 \%$ were admitted to an ICU, and $2 \%$ to $3 \%$ died. ${ }^{8}$ While this suggests that close to $70 \%$ to $80 \%$ of COVID-19 infections are mild and do not require hospitalization, this varied with age (and these data included patients 19 and younger). Hospitalization occurred in $14 \%$ to $21 \%$ of adults 20 to 44 years of age, $21 \%$ to $28 \%$ of adults 45 to 54 years of age, $21 \%$ to $30 \%$ of adults 55 to 64 years of age, 29\% to $44 \%$ of adults 65 to 74 years of age, $31 \%$ to $59 \%$ of adults 75 to 84 years of age, and $31 \%$ to $70 \%$ of adults 85 years of age and older. ${ }^{8}$ Thus, anywhere from $30 \%$ to $80 \%$ of adults with COVID-19 may have mild disease, depending on age.

There is little published information about the clinical characteristics of mild, non-pneumonia cases of COVID-19. The National Institutes of Health (NIH) in the United States recently defined mild COVID-19 illness as respiratory symptoms "without shortness of breath or abnormal imaging." Moderate illness is considered "evidence of lower respiratory disease by clinical assessment or imaging with $\mathrm{SpO}_{2}>93 \%$ on room air." ${ }^{\prime \prime}$ Patients
Sahana Kukan msc

with moderate or severe disease are recommended to be hospitalized, ${ }^{6,9}$ and most patients who have been hospitalized with COVID-19 will have pneumonia. ${ }^{3}$ Thus, the absence of pneumonia is key in differentiating between mild and moderate disease. Without imaging, family physicians will need to rule out pneumonia clinically during virtual assessments.

\section{Can the characteristics of pneumonia in adults admitted to hospital help inform virtual assessments?}

Of 1099 patients with confirmed cases of COVID-19 pneumonia admitted to more than 500 Chinese hospitals, $84 \%$ had mild cases and $16 \%$ had severe cases. ${ }^{3}$ Among patients with non-severe pneumonia, 57\% did not have a fever on admission, 33\% did not have a cough, and 85\% did not have shortness of breath. ${ }^{3}$ In $46 \%$ of the patients with mild pneumonia, the chest $\mathrm{x}$-ray was normal. ${ }^{3}$ Of 5700 patients with COVID-19 admitted to hospitals in New York City, only 31\% had a temperature above 38C, the respiratory rate was higher than 24 breaths per minute in only $17 \%$, and the heart rate was greater than or equal to 100 beats per minute in only $43 \%$ of patients. ${ }^{7}$ These are typical characteristics that have been proposed for use in virtual assessments, ${ }^{5}$ many of which may be normal in those who require hospitalization.

Even the symptoms of mild and severe pneumonia overlap broadly. Among the patients with mild pneumonia in the study of Chinese hospitals, typical symptoms on admission were cough (67\%), fever (43\%), fatigue (38\%), sputum (33\%), dyspnea (15\%), and myalgias (15\%). ${ }^{3}$ Among patients with severe pneumonia, their symptoms included cough (71\%), fever (48\%), fatigue (40\%), sputum (35\%), dyspnea (38\%), and myalgias (17\%). ${ }^{3}$ While chest exam findings were not reported, $84 \%$ of patients with mild pneumonia and $95 \%$ of patients with severe cases had abnormal chest CT findings. ${ }^{3}$ In virtual assessments, physicians may not be able to detect key symptoms in patients who would have radiologic features of mild or even severe COVID-19 pneumonia. Notably, the absence of dyspnea cannot be relied on to rule out pneumonia.

Oxygen saturation measurements have been proposed for inclusion in virtual assessments. ${ }^{5}$ In $50 \%$ of patients admitted to hospital in the New York City study, the oxygen saturation was $95 \% .^{7}$ The NIH definition of moderate disease includes an oxygen saturation greater than or equal to $93 \% .^{9}$ An oxygen saturation below $94 \%$ is considered severe pneumonia ${ }^{9}$ and is associated with increased mortality. ${ }^{10}$ This suggests a limited 
role for oxygen saturation in differentiating between mild and moderate disease. By the time oxygen saturation is declining, the disease may already be advanced and severe. Identifying pneumonia early may be critical. Mortality among patients with mild pneumonia admitted to hospital was $0.1 \%$ compared with $8.1 \%$ in patients with severe pneumonia in the Chinese study. ${ }^{3}$

\section{Are there validated tools that can rule out community-acquired pneumonia without the need for imaging?}

The ATS/IDSA 2019 guideline states that clinical signs and symptoms are known to be inaccurate for the diagnosis of community-acquired pneumonia in the absence of imaging. ${ }^{2}$ A previous American College of Chest Physicians guideline suggested the absence of abnormal vital signs and a clear chest examination could reduce the likelihood of pneumonia sufficiently that chest imaging was not needed. ${ }^{11}$ Abnormal vital signs included a heart rate greater than 100 beats per minute, a respiratory rate higher than 24 breaths per minute, and an oral temperature higher than 38C. ${ }^{11}$ A more recent systematic review also found that a normal pulmonary exam and the absence of abnormal vital signs could significantly reduce the likelihood of pneumonia being present. ${ }^{12}$

The negative likelihood ratio (LR-) for the absence of these findings was $0.10 .^{12}$ This means in younger adults (ages 20 to 64) with a prevalence of COVID-19 pneumonia between $20 \%$ and $30 \%,{ }^{8}$ the absence of these findings could reduce the risk that pneumonia is present to between $2.4 \%$ and $4 \%$. For older adults (65 and older) with a pneumonia prevalence of $30 \%$ to $50 \%,{ }^{8}$ the likelihood of pneumonia when all findings are absent would be between $4 \%$ and $9 \%$. However, these low risks would require a normal chest examination, which would not be part of a virtual assessment.

Where the absence of abnormal vital signs alone was used, the LR- was $0.24 .{ }^{12}$ Relying on this in virtual assessments would reduce the likelihood of pneumonia in younger adults to between $6 \%$ and $10 \%$. In older adults with a pneumonia prevalence of $30 \%$ to $50 \%$, normal vital signs could reduce the chance that pneumonia was present to between 10\% and 19\%. Given this risk for pneumonia and the high fatality rates in elderly patients, ${ }^{3,8}$ imaging would seem warranted even where vital signs are normal. In younger adults, the absence of these characteristics may reduce the need for a chest $x$-ray sufficiently, but accurate measurements of vital signs would be required to achieve this level of certainty.

\section{Can validated pneumonia severity ratings be used to identify adults with COVID-19 at low risk for mortality, such that even if pneumonia is missed, there is a low risk of death?}

The ATS/IDSA guideline recommends the use of the Pneumonia Severity Index/Patient Outcomes Research
Team (PSI/PORT) score to identify adults with low-, moderate-, and high-risk pneumonia. ${ }^{13}$ A PSI score lower than 70 is classified as low risk and appropriate for outpatient management, with a 30-day mortality less than $1 \%{ }^{14}$ However, even where adults had a PSI score higher than 70, indicating an elevated risk, the 30-day mortality remained below $1 \%$ if the following symptoms were absent: altered mental status, heart rate above 125 beats per minute, respiratory rate higher than 30 breaths per minute, systolic blood pressure below $90 \mathrm{~mm} \mathrm{Hg}$, and an oral temperature less than $35 \mathrm{C}$ or greater than $40 \mathrm{C} .{ }^{14}$ However, these mortality rates reflect what could be expected with outpatient antibiotic treatment. Low-risk adults with COVID-19 infections would not receive a specific treatment. Thus, the PSI mortality estimates may not apply in cases of community-acquired pneumonia associated with COVID-19.

\section{Are there any tools specific to COVID-19 for predicting disease severity or the need for admission?}

A recent systematic review examined 10 models for predicting a severe prognosis or the need for admission in COVID-19 patients. ${ }^{15}$ The authors concluded all the studies had serious flaws and the models' performance under actual clinical conditions was unknown. None were recommended for clinical use currently. Some models relied on information not available in virtual encounters, such as C-reactive protein and CT scoring. A similar conclusion was reached by an Oxford group after assessing systematic reviews and guidelines. ${ }^{16}$ At present, there are no validated tools specific to COVID-19 to assist family physicians in deciding whether communitybased care is appropriate for their patients. ${ }^{17}$

\section{Conclusion}

Assessing the risk for pneumonia in virtual assessments is critical for determining who requires hospital care and who does not. ${ }^{6,9}$ The NIH notes "given that pulmonary disease can rapidly progress, patients with moderate COVID-19 should be admitted to a health care facility."9 Given the risks when pneumonia is present, a low threshold for obtaining imaging is needed. This may be particularly important for older individuals in whom pneumonia symptoms may be atypical or absent, and among whom mortality is high. However, while mortality is highest among elderly patients, CDC data indicate that severe COVID-19 complications occur in adults of all ages. ${ }^{8}$

Family physicians need to be aware of the limitations of virtual assessments for suspected COVID-19. In-person assessments and imaging will be needed in many instances to allow for the early identification of pneumonia and appropriate hospital care.6,9 Whether these in-persons assessments should be provided in family practices, in emergency departments, or in established COVID-19 assessment centres is a further 
consideration. Clearly, research is urgently required to improve the evidence base for identifying mild cases of COVID-19 in community contexts. Relying on virtual assessments in the absence of valid clinical assessment tools and rigorous evaluations may do more harm than good if patients have severe pneumonia by the time they are referred to hospital.

Dr Mclsaac is a staff physician in the Ray D Wolfe Department of Family Medicine in the Sinai Health System in Toronto and an Associate Professor in the Department of Family and Community Medicine at the University of Toronto. Dr Upshur is a Professor in the Department of Family and Community Medicine and the Dalla Lana School of Public Health at the University of Toronto and Associate Director of the Lunenfeld-Tanenbaum Research Institute in the Sinai Health System in Toronto. Ms Kukan is a research coordinator working with Dr Mclsaac on grant-funded research in the Granovsky Gluskin Family Medicine Centre in the Sinai Health System in Toronto.

\section{Competing interests}

None declared

\section{Correspondence}

Dr Warren Mclsaac; email warren.mcisaac@sinaihealth.ca

The opinions expressed in commentaries are those of the authors. Publication does not imply endorsement by the College of Family Physicians of Canada.

\section{References}

1. Wu Z, McGoogan JM. Characteristics of and important lessons from the coronavirus disease 2019 (COVID-19) outbreak in China: summary of a report of 72314 cases from the Chinese Center for Disease Control and Prevention. JAMA 2020;323(13):1239-42.

2 Metlay JP, Waterer GW, Long AC, Anzueto A, Brozek J, Crothers K, et al. Diagnosis and treatment of Adults with Community Acquired Pneumonia: An official clinical practice guideline of the American Thoracic Society and Infectious Diseases Society of America. Am J Resp Crit Care Med 2019;200(7):e45-e67.

3. Guan WJ, Ni ZY, Hu Y, Liang WH, Ou CQ, He JX, et al. Clinical characteristics of coronavirus disease 2019 in China. NEJM 2020;382(18):1708-20.
4. Arsenault M, Evans B, Karanofsky K, Gardie J, Shulha M. Covid 19 - Practising Telemedicine in the pandemic [blog]. Can Fam Physician 2020 Mar 26. Available from: https://www.cfp.ca/news/2020/03/26/3-26-1.

5. Greenlagh $\mathrm{T}$, Koh $\mathrm{GCH}, \mathrm{Car}$ J. Covid-19: a remote assessment in primary care. BMJ 2020;368:m1182.

6. Gandhi RT, Lynch JB, del Rio C. Mild or moderate COVID-19. NEJM 2020 April 24.

7. Richardson S, Hirsch JS, Narasimhan M, Crawford JM, McGinn T, Davidson KW, et al. Presenting characteristics, comorbidities, and outcomes among 5700 patients hospitalized with COVID-19 in the New York City area. JAMA 2020;323(20):2052-59.

8. CDC COVID-19 Response Team. Severe outcomes among patients with coronavirus disease 2019 (COVID-19)-United States, February 12-March 16 2020. MMWR 2020;69(12):343-6.

9. COVID-19 Treatment Guidelines Panel. Coronavirus disease 2019 (COVID-19) treatment guidelines. National Institutes of Health. Available from: https://www. covid19treatmentguidelines.nih.gov/. Accessed 2020 May 08

10. Majumdar SR, Eurich DT, Gamble JM, Senthilselvan A, Marrie TJ. Oxygen saturation levels less than $92 \%$ are associated with major adverse events in outpatients with pneumonia: a population-based cohort study. Clin Infect Dis 2011;52(3):325-31.

11. Irwin RS, Baumann MH, Bolser DC, Boulet LP, Braman SS, Brightling CE, et al. Diagnosis and management of cough executive summary: ACCP evidence-based clinical practice guidelines. Chest 2005;129(1):1S-23S.

12. Marchello CS, Ebell MH, Dale AP, Harvill ET, Shen Y, Whalen CC. Signs and symptoms that rule out community-acquired pneumonia in outpatient adults: a systematic review and meta-analysis. JABFM 2019;32(2):234-47.

13. Olsen G, Davis AM. Diagnosis and treatment of community-acquired pneumonia. JAMA 2020;323(9):885-6.

14. Fine MJ, Auble TE, Yealy DM, Hanusa BH, Weissfeld LA, Singer DE, et al. A prediction rule to identify low-risk patients with community-acquired pneumonia. NEJM 1997;336(4):243-50.

15. Wynants L, Van Calster B, Riley RD, Heinze G, Schuit E, et al. Prediction models for diagnosis and prognosis of covid-19 infection: systematic review and critical appraisal. BMJ 2020;369:m1328.

16. Burrow R, Trewell J, Roberts N. What clinical features or scoring system, if any, might best predict a benefit from hospital admission for patients with COVID-19. Available from: https://www.cebm.net/covid-19/. Accessed 2020 May 7.

17. Sperrin M, Grant SW, Peek N. Prediction models for diagnosis and prognosis in Covid-19 [editorial]. BMJ 2020;369:m1464. 